
\title{
Delayed orthostatic hypotension in Parkinson's disease
}

\author{
Sang-Won Yoo $\mathbb{D}^{1}$, Joong-Seok Kim $\mathbb{D}^{1 凶}$, Ji-Yeon Yoo $\mathbb{D}^{1}$, Eunkyeong Yun $\mathbb{D}^{2}$, Uicheul Yoon $\mathbb{D}^{2}$, Na-Young Shin $\mathbb{D}^{3}$ and \\ Kwang-Soo Lee (iD)
}

Orthostatic hypotension $(\mathrm{OH})$ is relatively common in the early stage of Parkinson's disease (PD). It is divided into delayed OH and classical $\mathrm{OH}$. Classical $\mathrm{OH}$ in PD has been investigated widely, however, the clinical implications of delayed OH in PD have seldom been studied. The purpose of this study is to characterize delayed $\mathrm{OH}$ in PD. A total of 285 patients with early drug-naïve PD were enrolled and divided into three groups according to orthostatic change: $\mathrm{no}-\mathrm{OH}$, delayed $\mathrm{OH}$, and classical $\mathrm{OH}$. The disease severity in terms of motor, non-motor, and cognitive functions was assessed. The cortical thickness of 82 patients was analyzed with brain magnetic resonance imaging. The differences among groups and linear tendency in the order of no- $\mathrm{OH}$, delayed $\mathrm{OH}$, and classical $\mathrm{OH}$ were investigated. Seventy-seven patients were re-evaluated. Initial and follow-up evaluations were explored to discern any temporal effects of orthostasis on disease severity. Sixty-four (22.5\%) patients were defined as having delayed $\mathrm{OH}$ and 117 (41.1\%) had classical $\mathrm{OH}$. Between-group comparisons revealed that classical $\mathrm{OH}$ had the worst outcomes in motor, non-motor, cognitive, and cortical thickness, compared to the other groups. $\mathrm{No}-\mathrm{OH}$ and delayed $\mathrm{OH}$ did not differ significantly. Linear trends across the pre-ordered $\mathrm{OH}$ subtypes found that clinical parameters worsened along with the orthostatic challenge. Clinical scales deteriorated and the linear gradient was maintained during the follow-up period. This study suggests that delayed $\mathrm{OH}$ is a mild form of classical $\mathrm{OH}$ in PD. PD with delayed $\mathrm{OH}$ has milder disease severity and progression.

npj Parkinson's Disease (2021)7:37; https://doi.org/10.1038/s41531-021-00181-y

\section{INTRODUCTION}

Dysautonomia is a well-known non-motor feature that is discovered in the prodrome of Parkinson's disease (PD) ${ }^{1,2}$ Orthostatic hypotension $(\mathrm{OH})$ is found in early drug-naive $\mathrm{PD}^{3}$, and its presence is consistently associated with worse outcomes ${ }^{4-7}$.

$\mathrm{OH}$ is clinically divided into classical $\mathrm{OH}$ and delayed $\mathrm{OH}^{8,9}$. Classical $\mathrm{OH}$ is conventionally defined as sustained decrease in systolic blood pressure (SBP) $\geq 20 \mathrm{mmHg}$ and/or diastolic blood pressure (DBP) $10 \mathrm{mmHg}$ within $3 \mathrm{~min}$ of standing, and delayed $\mathrm{OH}$ is considered when the progressive blood pressure drop surpasses the margin of change after $3 \mathrm{~min}$. The clinical implications of delayed $\mathrm{OH}$ are suggested to be a non-benign, mild, or early form of sympathetic adrenergic dysfunction ${ }^{10}$. Although its significance was seldom studied in populations with PD, a longitudinal follow-up study revealed some individuals with delayed $\mathrm{OH}$ progressed to classical $\mathrm{OH}$ and developed asynucleinopathy ${ }^{11}$.

The assumption of present study was that delayed $\mathrm{OH}$ is a mild form of neurogenic $\mathrm{OH}$ in early $\mathrm{PD}$, representing milder disease severity. Patients were sub-grouped into a hypothesis-driven ordinal scale. The expected outcomes were between-group differences and a linear gradient across the subtypes, and results that were maintained with time. This would prove that patients with delayed $\mathrm{OH}$ have different motor and non-motor phenotypes and a distinct prognosis from other groups.

\section{RESULTS}

\section{Baseline characteristics}

The flow of participants is presented in Fig. 1 and the baseline demographics of PD patients are summarized in Table 1. The mean age was $69.6 \pm 9.3$ years old and 132 (46.3\%) were female.
The median duration of disease was 1 (1.0) year (interquartile range, IQR). The mean total sum of Unified Parkinson's Disease Rating Scale (UPDRS) was $23.3 \pm 12.6$ with a median modified Hoehn and Yahr (H\&Y) of 2.0 (IQR, 1.0). The mean score of MiniMental Status Examination (MMSE) was $27.0 \pm 2.8$ and the median Clinical Dementia Rating (CDR) was 0.5 (IQR, 0). Sixty-four patients (22.5\%) were defined to have delayed $\mathrm{OH}$ and $117(41.1 \%)$ had classical $\mathrm{OH}$. The mean uptake of delayed heart-to-mediastinum $(\mathrm{H} / \mathrm{M})$ ratio was $1.55 \pm 0.37$. Classical $\mathrm{OH}$ were older than other groups.

\section{Comparisons across orthostatic hypotension groups}

Between-group comparisons, adjusted for age, sex, and disease duration were analyzed and encapsulated in Table 2 and Supplementary Table 1. Classical $\mathrm{OH}$ scored higher in UPDRS Part II (no-OH vs. delayed $\mathrm{OH}$ vs. classical $\mathrm{OH}: 5.2 \pm 0.4$ vs. $5.1 \pm 0.5$ vs. $6.8 \pm 0.4 ; p=0.005 ; a=b<c$ ), Part III (no-OH vs. delayed $\mathrm{OH}$ vs. classical $\mathrm{OH}: 14.9 \pm 0.8$ vs. $13.9 \pm 1.1$ vs $17.9 \pm 0.8 ; p=0.008 ; a=b$ $<c$ ), and UPDRS total score (no-OH vs. delayed $\mathrm{OH}$ vs. classical $\mathrm{OH}$ : $21.4 \pm 1.1$ vs. $20.7 \pm 1.5$ vs. $26.4 \pm 1.1 ; p=0.002 ; a<c$ ). The classical $\mathrm{OH}$ group also had higher supine systolic blood pressure than the no-OH group. Of 285 PD, 154 (54.0\%) had nocturnal hypertension and 138 (48.4\%) were non-dippers. UPDRS scores, supine blood pressure (BP), the proportions of nocturnal hypertension, and nondipper all showed an ascending linear trend across the $\mathrm{OH}$ groups.

Classical $\mathrm{OH}$ had higher Non-Motor Symptoms Scale (NMSS) scores (no-OH vs. classical $\mathrm{OH}: 30.2 \pm 4.5$ vs. $47.1 \pm 4.6 ; p=0.032$ ), $\mathrm{OHQ}$ Part II (no-OH vs. classical $\mathrm{OH}$ : $4.7 \pm 1.1$ vs. $8.7 \pm 1.1 ; p=$ 0.026), and Rapid-eye-movement Sleep Behavior Disorder Screening Questionnaire (RBDSQ; no-OH vs. classical $\mathrm{OH}: 2.5 \pm 0.3$ vs. $3.8 \pm 0.3 ; p=0.025)$ than no-OH. A positive linear trend was found across the groups in the NMSS, Orthostatic Hypotension

\footnotetext{
Department of Neurology, College of Medicine, The Catholic University of Korea, Seoul, Republic of Korea. ${ }^{2}$ Department of Biomedical Engineering, College of Health and Medical Science, Catholic University of Daegu, Gyeongbuk, Republic of Korea. ${ }^{3}$ Department of Radiology, College of Medicine, The Catholic University of Korea, Seoul, Republic of Korea. ${ }^{凶}$ email: neuronet@catholic.ac.kr
} 
Questionnaire (OHQ) Part II, Montgomery-Asberg Depression Rating Scale (MADRS), and RBDSQ questionnaires.

Cortical thickness comparison also depicted a similar pattern of between-group differences, when controlled for age, sex, disease duration, and MMSE. The whole cerebral cortex (no-OH vs. classical $\mathrm{OH}: 3.27 \pm 0.02$ vs. $3.15 \pm 0.03 ; p=0.002$ ), in particular the frontal and parietal lobes (no-OH vs. classical $\mathrm{OH}: 3.17 \pm 0.02$ vs. $3.05 \pm 0.02, p=0.001 ; 2.98 \pm 0.03$ vs. $2.79 \pm 0.05, p=0.007$; respectively), manifested thinner thickness in patients with

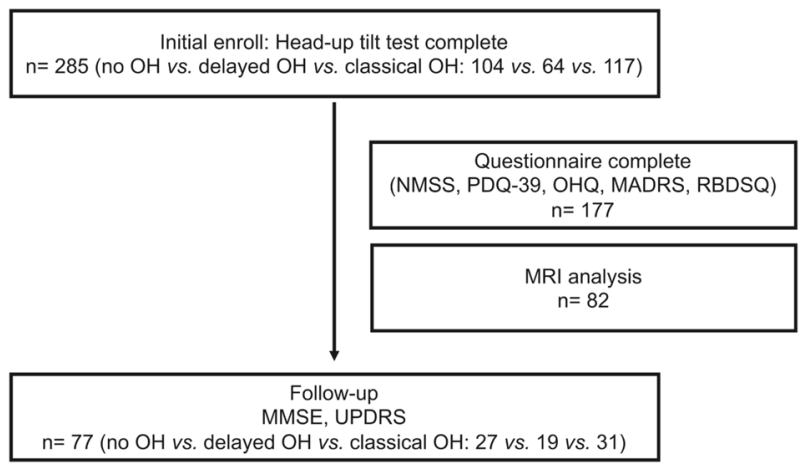

Fig. 1 Enrolled patients study flow. NMSS Non-Motor Symptoms Scale, PDQ-39 Parkinson's Disease Quality of Life-39, OHQ Orthostatic Hypotension Questionnaire, MADRS Montgomery-Asberg Depression Rating Scale, RBDSQ REM Sleep Behavior Disorder Screening Questionnaire, UPDRS Unified Parkinson's Disease Rating Scale, MMSE Mini-Mental Status Examination. classical $\mathrm{OH}$ group compared to no-OH. There was a negative linear tendency in the general cortex, except the left temporal and occipital cortex, across the $\mathrm{OH}$ groups (Table 3).

\section{Longitudinal influences of orthostatic hypotension groups}

Seventy-seven PD patients were followed for a mean period of $24.2 \pm 5.3$ months (no-OH vs. delayed $\mathrm{OH}$ vs. classical $\mathrm{OH}: 23.3 \pm$ 5.1 vs. $23.0 \pm 4.8$ vs. $25.7 \pm 5.5 ; p=0.117$ ) and the longitudinal influences of the orthostatic BP drop are presented in Table 4 and Fig. 2. In this sub-analysis, age, disease duration and levodopa equivalent daily dose (LEDD) of the last trace point did not differ across the groups (age: $p=0.065$; disease duration: $p=0.531$; LEDD: $p=0.137)$. UPDRS Part II and MMSE score demonstrated progression during the follow-up period (time effects: $p=0.019, p$ $=0.008$; respectively) and classical $\mathrm{OH}$ had worse outcomes on these scales (group effects: $p=0.006, p=0.006 ; a=b<c, a>c$; respectively). UPDRS total sum and Part III did not display any impairment during the follow-up (time effects: $p=0.075, p=$ 0.440; respectively), but maintained between-group differences (group effects: $p=0.004, p=0.016$; respectively). There were no interactions between time and group effects among the analyzed scales. When dopaminergic treatment effects were adjusted, UPDRS scores and MMSE did not worsen significantly over the follow-up period (time effect: $p>0.05$ ). Classical $\mathrm{OH}$ similarly maintained its worsening trend on UPDRS total sum, Part II, Part III and MMSE scores (group effects: $p=0.012, p=0.023, p=0.034, p$ $=0.006$; interaction: $p=0.574, p=0.872, p=0.346 ; p=0.874$; Supplementary Table 2 and Supplementary Fig. 1).

Table 1. Clinical characteristics.

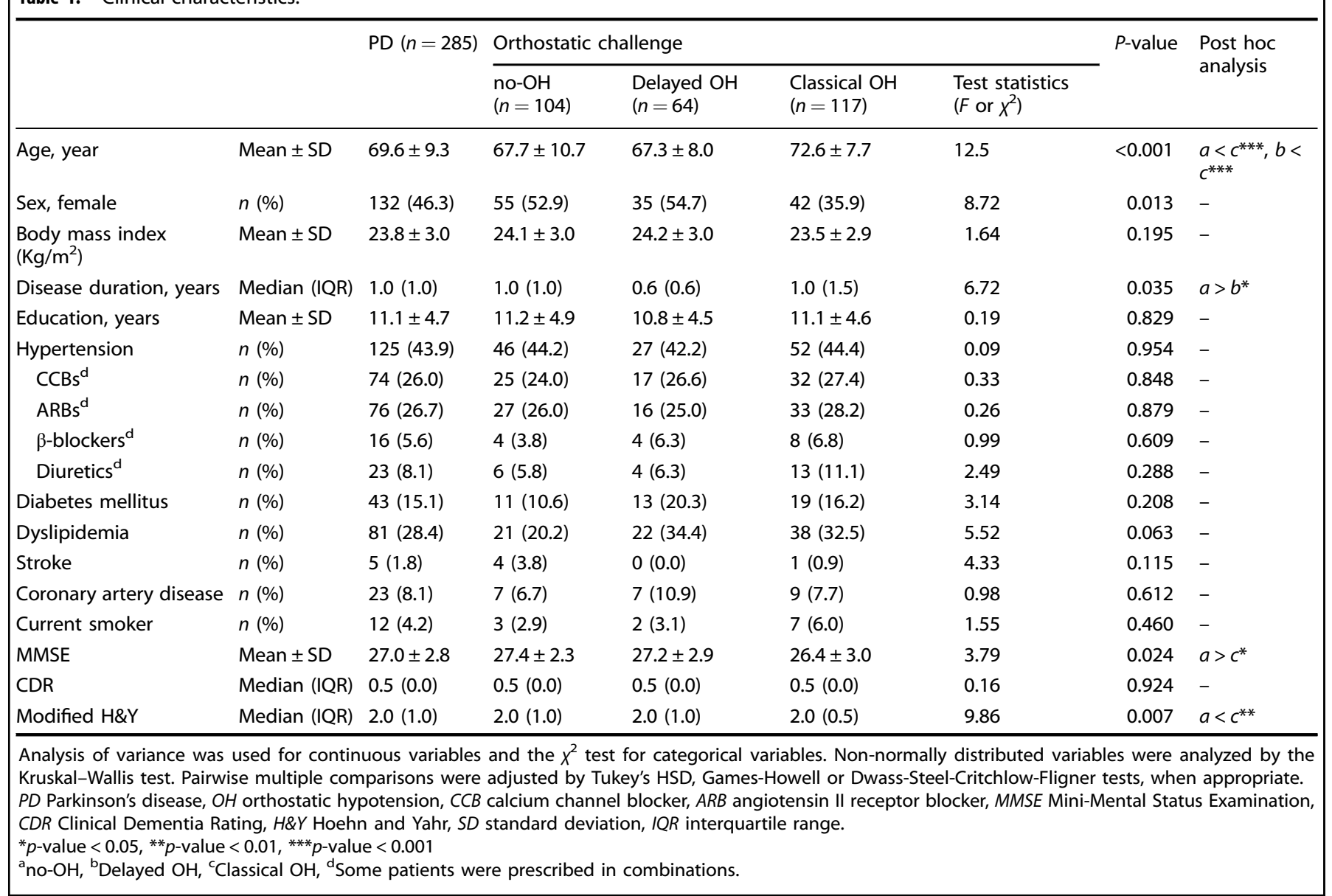




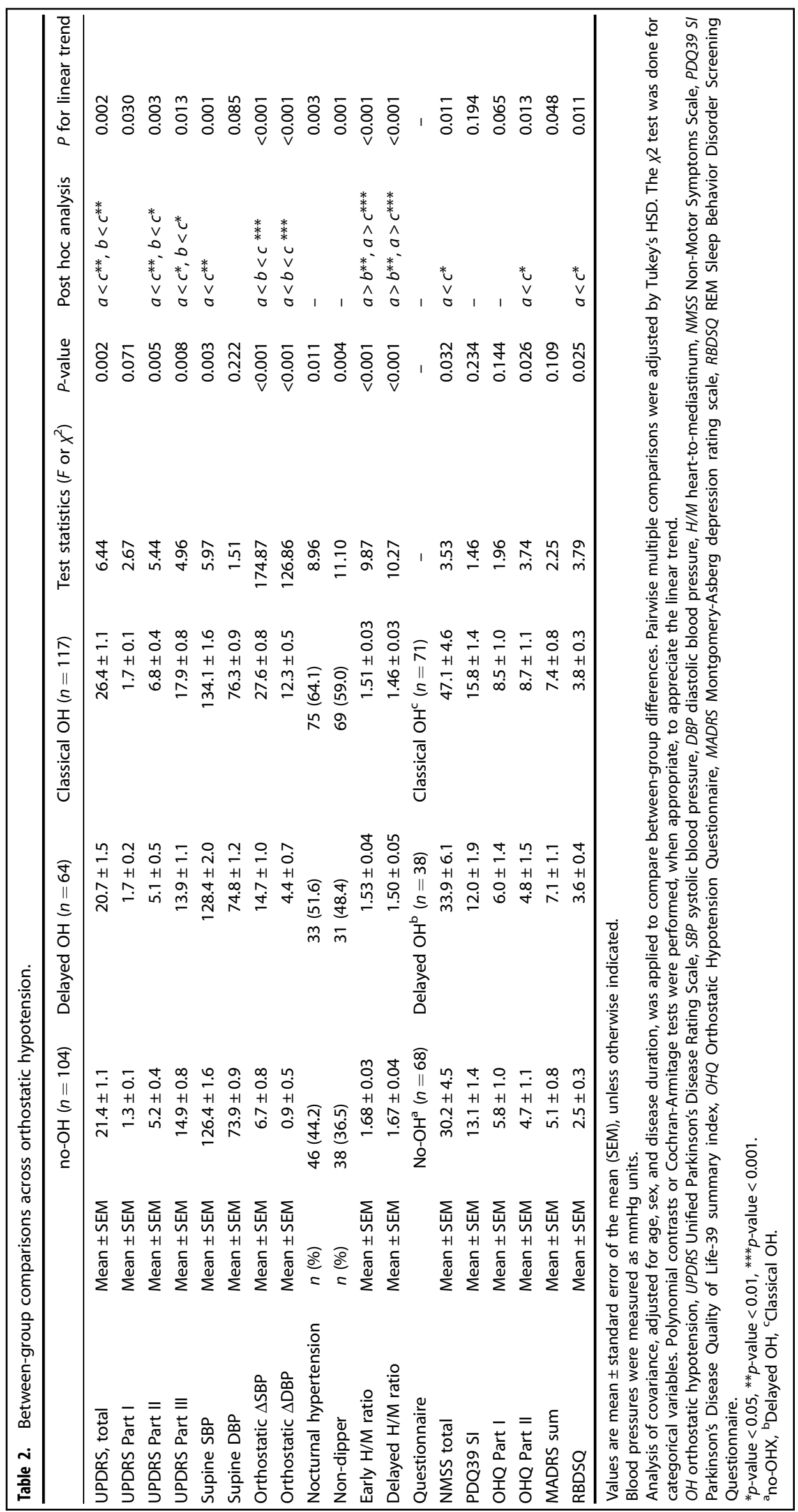


4

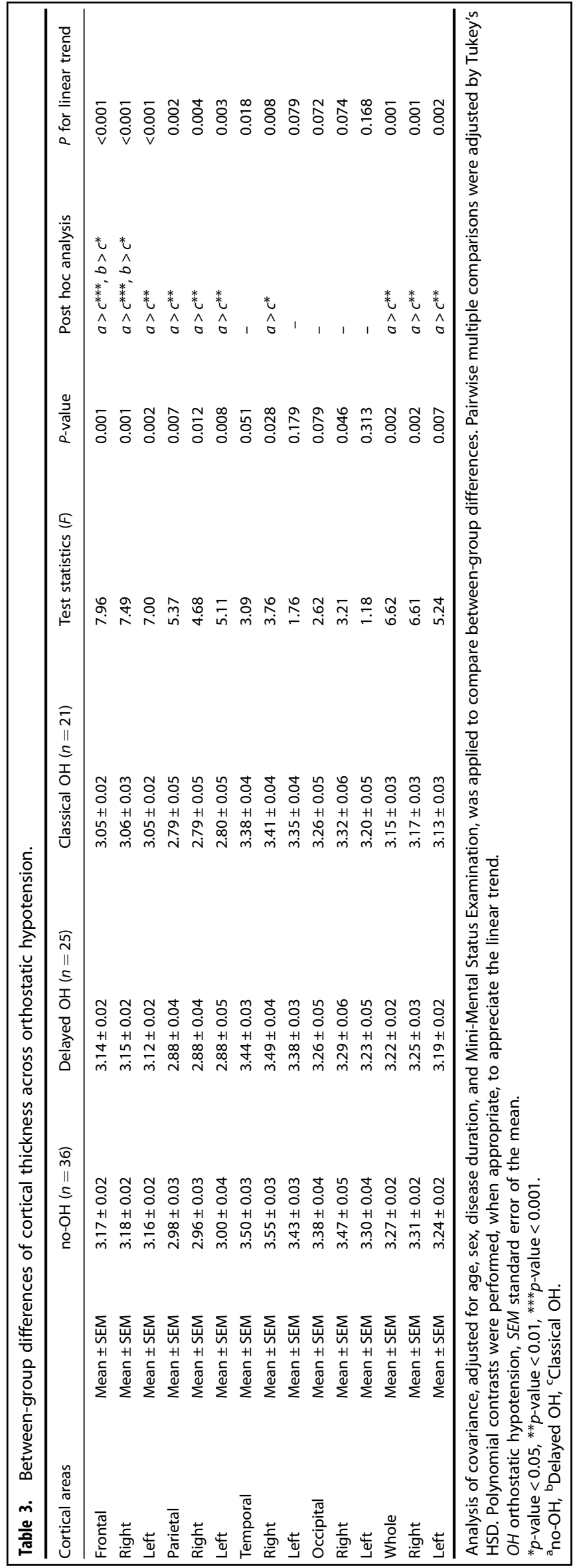

\section{DISCUSSION}

In this study, ordinal subtypes of $\mathrm{OH}$ (delayed $\mathrm{OH}$ vs. classical $\mathrm{OH}$ ) were compared to investigate the nature of $\mathrm{OH}$ in early PD. Group with classical $\mathrm{OH}$ manifested more severe clinical scales and biomarkers than other groups. However, no- $\mathrm{OH}$ and delayed $\mathrm{OH}$ groups did not differ significantly. The disparities had a linear gradient pattern across the $\mathrm{OH}$ spectrum. These findings were sustained during the longitudinal follow-up.

The population of this study was in the early stage of disease with non-demented mild PD. The similar clinical status reflects a relatively homogeneous pathophysiologic stage confined to the brainstem according to 'bottom-up' theory ${ }^{12,13}$. PD patients with classical $\mathrm{OH}$ were older than other groups, and the prevalence of classical $\mathrm{OH}$ was similar when compared to previous studies that estimated it in a range of $14-54 \%{ }^{3}$.

The prevalence of delayed $\mathrm{OH}$ was also comparable ${ }^{11}$, but with a different clinical context. Gibbons and Freeman explored the natural history of delayed $\mathrm{OH}$ in a 10-year follow-up study and observed that about half progressed to classical $\mathrm{OH}$. Among the subjects with delayed $\mathrm{OH}$ in initial testing who progressed to classical $\mathrm{OH},>50 \%$ developed a-synucleinopathy. This study was not designed to investigate delayed $\mathrm{OH}$ in PD; therefore, it was difficult to infer any clinical significance in PD population. Our research is of value that it studied delayed $\mathrm{OH}$ in PD.

Motor related (dopaminergic) functions, including daily activities, were preferentially more affected in PD with classical $\mathrm{OH}$ when the covariates were controlled. Motor features of daily activities worsened over a 2-year period, in line with a previous study ${ }^{14}$. The differences among groups and aggravating linear trend of disease severity across groups were maintained throughout the follow-up period. The potential causes of the motor disparities could be the result of severer underlying disease burden and/or be attributed to fatigue or end-organ damage by blood pressure instability ${ }^{15,16}$.

Non-motor features were found to be more severe in PD with classical $\mathrm{OH}$. Worse non-motor features could have affected adverse motor outcomes ${ }^{17}$. The participants showed increasing non-motor severity across the $\mathrm{OH}$ groups. The positive association of nondopaminergic parameters with ordinal $\mathrm{OH}$ further strengthens the argument that delayed $\mathrm{OH}$ is a mild form of neurogenic $\mathrm{OH}$ in early PD. The cognition of all three groups worsened with time. $\mathrm{PD}$ with classical $\mathrm{OH}$ progressed more aggressively while the worsening of no-OH and delayed $\mathrm{OH}$ were not disparate. This result is consistent with a previous study where $\mathrm{OH}$ is reported to impact cognitive decline $e^{5,18}$.

After adjusting for dopaminergic therapy, the cognitive and motor severities did not demonstrate significant worsening, regardless of the $\mathrm{OH}$ types. This was anticipatory because early non-demented PD (motor and cognitive aspects) responded to dopaminergic replacement ${ }^{19}$. However, classical $\mathrm{OH}$ had worse overall severities than the other groups, and its unfavorable outcomes were sustained during the follow-up period, independent of dopaminergic treatment. The observed baseline betweengroup differences and worsening tendencies across the $\mathrm{OH}$ spectrum during the follow-up period argues that different levels of orthostatic challenge may affect distinctive clinical progressions.

Association between $\mathrm{OH}$ and cognitive impairment has been discussed previously. In this cohort of early de novo PD, noradrenergic deficiency by locus coeruleus impairment and vascular theory of cerebral hypoperfusion could have attributed to the more rapid decline of cognition in classical $\mathrm{OH}^{20,21}$. Cortical thinning could also be a cause of such a finding.

Biomarkers of the cardiovascular system and cortical thickness depicted similar patterns across the groups as the clinical parameters. The frequency of disrupted circadian blood pressures, assessed by 24-hour ambulatory blood pressure monitoring, 


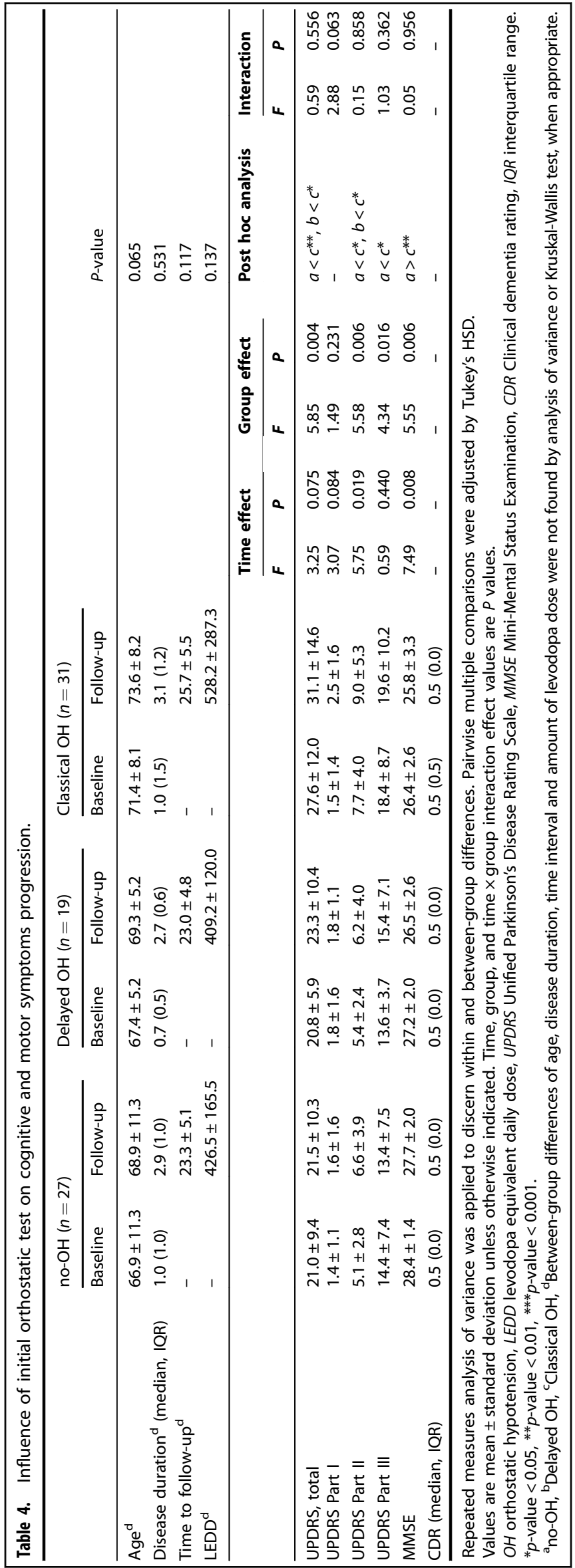

increased with the planned order of $\mathrm{OH}$ types. Patients with $\mathrm{OH}$ (both classical or delayed) were more aggressively deprived of cardiac sympathetic innervation than those with no-OH. Supine blood pressure tended to increase across the $\mathrm{OH}$ spectrum. The worsening gradient across the groups represented more damaged arterial baroreflex abnormalities'. Anterior dominant cortical thinning was worst in the classical $\mathrm{OH}$. This was replicated in a previous study with hypotensive insults as a causative mechanism $^{22-25}$. The cortex tended to become thinner across the $\mathrm{OH}$ groups which may result from different levels of hypotensive insults.

The strength of this study was that a large number of early drugnaïve PD were enrolled with extensive assessments encompassing non-motor and motor features. Other studies with comparable early PD involved small populations ${ }^{26,27}$. Only recently, a large scale prospective cohort was published however this performed limited evaluations and provided limited information ${ }^{3}$. PD is an agedependent neurodegenerative disease in which clinical parameters are affected by aging. In this study, baseline characteristics with demonstrated differences were re-assessed with an adjustment of covariates, unlike in previous studies ${ }^{3}$. This study has several limitations. First, the enrolled patients did not go through every measurement due to the condition of each patient, and they were excluded from sub-analyses. In addition, many patients were lost during the follow-up. The large drop-outs are the major weakness because this would inevitably deepen selection bias. However, the study population included mild non-demented de novo PD to ensure reasonably homogenous pathologic stages and was followed for 2-year period on average. Its homogeneity of mild disease severity and relatively short follow-up duration may mitigate the bias that only the fitter patients could have been investigated. The large drop-outs could reduce the statistical power to discern group differences. On the contrary, it supports that achieved differences overcame the conservative null hypothesis produced by diminished statistical power; however, careful consideration is required in the interpretation since the drop-outs could also bias the alternative hypothesis. Second, autonomic evaluations were not comprehensive. Further evaluations of parasympathetic outflows could be of benefit in elucidating the pathophysiology of delayed $\mathrm{OH}$. Third, patients had comorbid diseases, such as hypertension and diabetes mellitus, which contribute to autonomic disturbances. In addition, many subjects with comorbid diseases were on medications. We did not analyze the interactive influence of systemic diseases or drugs that might play a pathophysiologic role in autonomic failure. Finally, the follow-up duration was too short for definite conclusiveness. Large prolonged prospective studies with comprehensive evaluations of clinical aspects and biomarkers are required to further describe delayed $\mathrm{OH}$.

In summary, not only the pattern of between-group differences but also the linear gradient across the ordinal subtypes of $\mathrm{OH}$, and the maintenance of such traits with time suggest that delayed $\mathrm{OH}$ is a mild form of classical neurogenic $\mathrm{OH}$ in early PD, associated with less severe clinical burden and deterioration. This may facilitate patient selections in future neuroprotective studies.

\section{METHODS}

\section{Participants}

This longitudinal study was approved by the Institutional Review at Seoul St. Mary's Hospital, and all subjects provided written informed consent to participate. All experiments were performed in accordance with relevant guidelines and regulations. The study was registered (Identification Number: KCT0005552) in the Clinical Research Information Service (CRIS; http://cris.nih.go.kr), which is an online clinical trial registration system established by the Korea Centers for Disease Control and Prevention (KCDC) with support from the Korea Ministry of Health and Welfare $(\mathrm{KMOHW})$ and embodied as a part of the Primary Registries in the World Health Organization (WHO) Registry Network. 

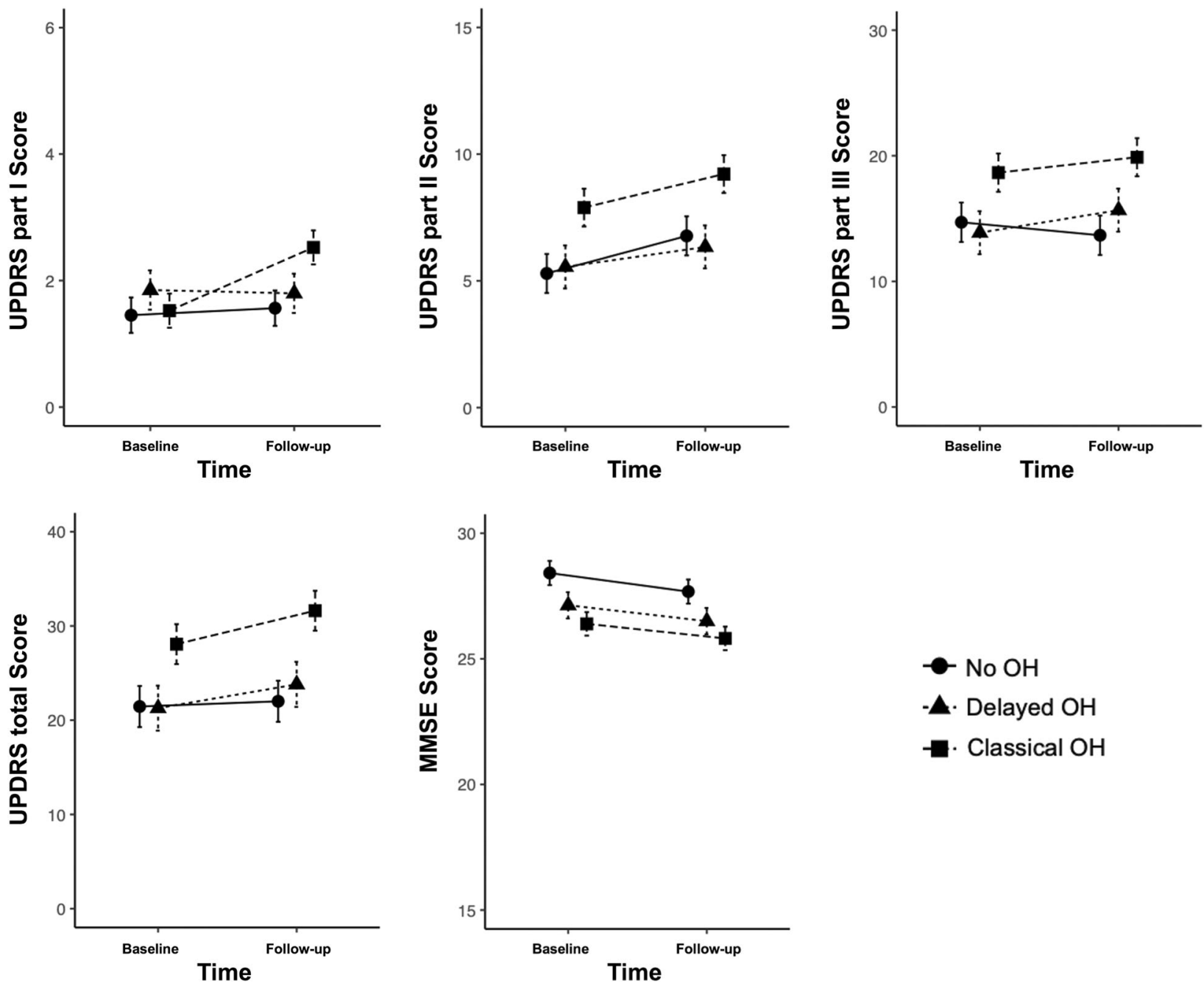

Fig. 2 Temporal progression of cognitive and motor severities across the groups. Error bars: standard error of the mean. UPDRS Unified Parkinson's Disease Rating Scale, MMSE Mini-Mental Status Examination, $\mathrm{OH}$ orthostatic hypotension.

Two hundred eighty-five drug-naïve patients newly diagnosed with PD between October 2014 and December 2019 were enrolled. The diagnosis of PD was based on the UK Parkinson's Disease Society Brain Bank clinical diagnostic criteria ${ }^{28}$, and its diagnosis was substantiated by positron emission tomography imaging studies using ${ }^{18} \mathrm{~F}-\mathrm{N}$-(3-fluoropropyl)-2beta-carbon ethoxy-3beta-(4-iodophenyl) nortropane and ${ }^{123}$-metaiodobenzylguanidine ${ }^{(23}$ I-MIBG) scintigraphy ${ }^{29,30}$. Baseline demographics such as age, sex, body mass index (BMI), disease duration, education, smoking status, and history of hypertension, diabetes mellitus, dyslipidemia, stroke, and coronary artery disease were investigated. Disease severity was evaluated with the UPDRS and modified H\&Y stage. Global cognition was assessed by MMSE and CDR.

Patients with any of the following indications were excluded from the initial enrollment: (1) any symptoms or signs of atypical and/or secondary parkinsonism, (2) documentation of atrial fibrillation during head-up tilt electrocardiographic monitoring, (3) history of diabetic neuropathy, (4) history of peripheral arterial disease, (5) history of symptomatic stroke that might affect cognition and general performance, (6) taking medications such as tricyclic antidepressant and alpha-adrenergic antagonists that influence orthostatic challenge, (7) family history of dementia, and (8) clinical suspicion of dementia $(C D R \geq 1)^{31}$.

The global cognitive efficiency and disease severity of 77 patients with PD were re-evaluated by using MMSE and UPDRS, respectively (Fig. 1).

\section{Questionnaires}

Questionnaires were completed by 177 patients. Non-motor features, mood, quality of life, parasomnia, and symptoms related to orthostatic challenge were evaluated with the NMSS, MADRS, Parkinson's Disease
Quality of Life-39 (PDQ-39), RBDSQ and OHQ, respectively ${ }^{32-36}$. The sums of each questionnaire were used for analyses.

\section{Head-up tilt test}

All patients were at a full resting state before the exam. Continuous electrocardiograph leads and non-invasive blood pressure monitoring equipment were applied to the patients (YM6000, Mediana Tech, Redmond, WA, USA). A supine position was maintained for 20 min before tilting to $60^{\circ}$ (Enraf-Nonius, Rotterdam, The Netherlands). While in the supine position, blood pressure and heart rate were measured every $5 \mathrm{~min}$ for $20 \mathrm{~min}$. At the $60^{\circ}$ position, blood pressure and heart rate were measured at $0,3,5,10,15$, and $20 \mathrm{~min}$. For analyses, the lowest tilt values for BP were chosen from 0 to $3 \mathrm{~min}$ for classical $\mathrm{OH}$, and from 5 to $20 \mathrm{~min}$ for delayed $\mathrm{OH}$. The first supine blood pressure (at $0 \mathrm{~min}$ ) was excluded, and maximal supine systolic and diastolic blood pressures were selected among the measurements at 5, 10, 15 , and $20 \mathrm{~min}$. The lowest systolic and diastolic values at 0 or $3 \mathrm{~min}$ during the tilted position were chosen. The orthostatic blood pressure changes of systole $(\triangle \mathrm{SBP})$ and diastole $(\triangle \mathrm{DBP})$ were calculated. Patients were categorized as having classical $\mathrm{OH}$ or delayed $\mathrm{OH}$ if $\triangle \mathrm{SBP}$ and/or $\triangle \mathrm{DBP} \geq 20 / 10 \mathrm{mmHg}$ within $3 \mathrm{~min}$ or when the BP drops occurred after $5 \mathrm{~min}^{8}$. Cases that satisfied both classical $\mathrm{OH}$ and delayed $\mathrm{OH}$ criteria were categorized as classical $\mathrm{OH}$.

\section{Twenty-four-hour ambulatory blood pressure monitoring}

Automated 24-hour blood pressure equipment (Mobil-O-Graph NG, IEM, Stolberg, Germany) was used to measure daytime and nighttime blood pressures from the upper arm. Blood pressures were recorded at 15-minute 
intervals throughout the day and 30-min intervals at night. Daytime was defined as a period between 8:00 a.m. and 23:59 p.m., and nighttime was from 00:00 a.m. to 07:59 a.m. Nocturnal hypertension was defined as increased absolute values of nighttime systolic and/or diastolic $B P \geq 120$ / $70 \mathrm{mmHg}^{37}$. Patients were classified as non-dipper if the ratio of night/day systolic and/or diastolic $\mathrm{BP} \geq 1^{38}$.

\section{I-metaiodobenzylguanidine scintigraphy}

${ }^{123}$ I-MIBG scintigraphy was performed using a dual-head camera equipped with a low-energy high-resolution collimator (Siemens), and data were collected at 30-min (early) and 2-h (delayed) time points after a $111 \mathrm{MBq}$ ${ }^{123}$ I-MIBG injection. A static image was obtained with a $128 \times 128$ matrix. Regions of interest were manually drawn around the heart and mediastinum. Tracer uptake was measured within each region of interest. For each time point, tracer uptake ratios of the $\mathrm{H} / \mathrm{M}$ ratio were calculated and defined as early $\mathrm{H} / \mathrm{M}(30 \mathrm{~min})$ and delayed $\mathrm{H} / \mathrm{M}(120 \mathrm{~min})$. The lower limits of the reference value for early and delayed $\mathrm{H} / \mathrm{M}$ ratios were set to be 1.70 and 1.78 , respectively ${ }^{30}$.

\section{Magnetic resonance imaging acquisition and cortical thickness measurements}

The cortical thickness of 82 patients was analyzed by brain magnetic resonance imaging (MRI). A 3D T1-weighted magnetization-prepared rapid gradient-echo sequence was acquired using a 3-T scanner (Magnetom Verio, Siemens Healthcare, Erlangen, Germany) with a 12-channel head coil. Parameters were as follows: sagittal acquisition with $\mathrm{FOV}=256 \times$ $256 \mathrm{~mm}^{2}$; voxel size $=1 \times 1 \times 1 \mathrm{~mm}^{3} ; \mathrm{TR}=1780 \mathrm{msec}$; $\mathrm{TE}=2.2 \mathrm{msec}$; flip angle $=9^{\circ}$; total acquisition time $=6 \mathrm{~min} 38 \mathrm{~s}$. For the measurement of cortical thickness, we used the CIVET pipeline (http://mcin.ca/civet/) as described in detail elsewhere ${ }^{39,40}$. The native T1-weighted images of each subject were corrected for intensity inhomogeneity and spatially normalized to the MNI-152 template. After that, the images were tissue classified and hemispheric inner and outer cortical surfaces were automatically extracted using the constrained Laplacian-based automated segmentation with the proximities algorithm. Cortical thickness was measured by calculating the Euclidean distance between corresponding vertices on the gray matter/cerebrospinal fluid intersection surface and the white matter/ gray matter boundary surface ${ }^{41}$. Diffusion smoothing with a $30-\mathrm{mm}$ full width at half maximum kernel (FWHM) was used to increase the signal-tonoise ratio. To extract the mean regional cortical thickness, we used the Automated Anatomical Labeling (AAL) atlas template to define regional boundaries and averaged the cortical thickness of vertices within each of the regions of interest for each subject. Less than one third of the study population was included due to alterations of acquisition protocol and MRI equipment, and only patients with suitable uniformity for analyses were selected.

\section{Statistical analysis}

All statistical analyses were performed with jamovi software (version 1.2.16; retrieved from https://www.jamovi.org) and $\mathrm{R}$ software with additional car and emmeans packages (version 3.6.3; retrieved from https://cran.r-project. org) for Mac. Descriptive analyses and the analysis of variance or Kruskal-Wallis tests were applied to describe the baseline characteristics of PD patients. Categorical variables were analyzed by the $x^{2}$ test. Subgroups of PD patients were examined by analysis of covariance, adjusted for age, sex, disease duration, and additional covariates when needed, to investigate between-group differences. To discern any linear gradient in the order of no- $\mathrm{OH}$, delayed $\mathrm{OH}$, and classical $\mathrm{OH}$, polynomial contrasts or Cochran-Armitage tests were used, as appropriate. In a subgroup analysis of PD patients, repeated measures analysis of variance was applied to measure the temporal patterns of PD subgroups. Multiple comparisons were corrected with a defined significance at a two-tailed $p$ value $<0.05$

\section{Reporting summary}

Further information on research design is available in the Nature Research Reporting Summary linked to this article.

\section{DATA AVAILABILITY}

Anonymized data generated during the current study are available from the corresponding author on reasonable request from individuals affiliated with research or health care institutions.

\section{CODE AVAILABILITY}

Jamovi is a statistical spreadsheet and graphical user interface (GUI) for R. All the analyses, except Cochran-Armitage, were performed basically using jamovi software as mentioned in the method, statistical analysis section. The $\mathrm{R}$ packages mentioned were utilized within jamovi GUI.

Received: 16 August 2020; Accepted: 26 February 2021; Published online: 14 April 2021

\section{REFERENCES}

1. Jain, S. \& Goldstein, D. S. Cardiovascular dysautonomia in Parkinson disease: from pathophysiology to pathogenesis. Neurobiol. Dis. 46, 572-580 (2012).

2. Pont-Sunyer, $C$. et al. The onset of nonmotor symptoms in Parkinson's disease (The ONSET PD Study). Mov. Disord. 30, 229-237 (2015).

3. Hiorth, Y. H., Pedersen, K. F., Dalen, I., Tysnes, O. B. \& Alves, G. Orthostatic hypotension in Parkinson disease: a 7-year prospective population-based study. Neurology 93, e1526-e1534 (2019).

4. Kim, J. S. et al. Association of cognitive dysfunction with neurocirculatory abnormalities in early Parkinson disease. Neurology 79, 1323-1331 (2012).

5. Anang, J. B. et al. Predictors of dementia in Parkinson disease: a prospective cohort study. Neurology 83, 1253-1260 (2014).

6. Fereshtehnejad, S. M. et al. New clinical subtypes of Parkinson disease and their longitudinal progression: a prospective cohort comparison with other phenotypes. JAMA Neurol. 72, 863-873 (2015).

7. De Pablo-Fernandez, E. et al. Association of autonomic dysfunction with disease progression and survival in Parkinson disease. JAMA Neurol. 74, 970-976 (2017).

8. Freeman, R. et al. Consensus statement on the definition of orthostatic hypotension, neurally mediated syncope and the postural tachycardia syndrome. Clin. Auton. Res. 21, 69-72 (2011).

9. Brignole, M. et al. Practical instructions for the 2018 ESC guidelines for the diagnosis and management of syncope. Eur. Heart J. 39, e43-e80 (2018).

10. Gibbons, C. H. \& Freeman, R. Delayed orthostatic hypotension: a frequent cause of orthostatic intolerance. Neurology 67, 28-32 (2006).

11. Gibbons, C. H. \& Freeman, R. Clinical implications of delayed orthostatic hypotension: A 10-year follow-up study. Neurology 85, 1362-1367 (2015).

12. Braak, H. et al. Staging of brain pathology related to sporadic Parkinson's disease. Neurobiol. Aging 24, 197-211 (2003).

13. Beach, T. G. et al. Unified staging system for Lewy body disorders: correlation with nigrostriatal degeneration, cognitive impairment and motor dysfunction. Acta Neuropathol. 117, 613-634 (2009).

14. Kotagal, V., Lineback, C., Bohnen, N. I. \& Albin, R. L. Orthostatic hypotension predicts motor decline in early Parkinson disease. Parkinsonism Relat. Disord. 32, 127-129 (2016).

15. Nakamura, T., Suzuki, M., Ueda, M., Hirayama, M. \& Katsuno, M. Lower body mass index is associated with orthostatic hypotension in Parkinson's disease. J. Neurol. Sci. 372, 14-18 (2017).

16. Kotagal, V., Szpara, A., Albin, R. L. \& Bohnen, N. I. Fatigue in Parkinson's disease associates with lower ambulatory diastolic blood pressure. J. Parkinsons Dis. 9, 575-581 (2019).

17. van der Heeden, J. F., Marinus, J., Martinez-Martin, P. \& van Hilten, J. J. Importance of nondopaminergic features in evaluating disease severity of Parkinson disease. Neurology 82, 412-418 (2014).

18. Pilotto, A. et al. Orthostatic hypotension and REM sleep behaviour disorder: impact on clinical outcomes in alpha-synucleinopathies. J. Neurol. Neurosurg. Psychiatry 90, 1257-1263 (2019).

19. Kehagia, A. A., Baker, R. A. \& Robbins, T. W. Cognitive impairment in Parkinson's disease: the dual syndrome hypothesis. Neurodegener. Dis. 11, 79-92 (2013).

20. McDonald, C., Newton, J. L. \& Burn, D. J. Orthostatic hypotension and cognitive impairment in Parkinson's disease: causation or association? Mov. Disord. 31 937-946 (2016).

21. Yoo, S. W. et al. Intervening effects of orthostatic blood pressure change on subcortical atrophy and cognition in de novo and drug-naïve Parkinson's disease. J. Parkinsons Dis. 10, 153-160 (2020).

22. Shin, K. J. et al. Cortical morphology in patients with orthostatic intolerance. Eur. Neurol. 73, 264-270 (2015). 
23. de la Torre, J. C. Critically attained threshold of cerebral hypoperfusion: the CATCH hypothesis of Alzheimer's pathogenesis. Neurobiol. Aging 21, 331-342 (2000).

24. Jochemsen, H. M. et al. Blood pressure and progression of brain atrophy: the SMART-MR Study. JAMA Neurol. 70, 1046-1053 (2013).

25. Zonneveld, $\mathrm{H}$. I. et al. The bidirectional association between reduced cerebral blood flow and brain atrophy in the general population. J. Cereb. Blood Flow. Metab. 35, 1882-1887 (2015).

26. Bonuccelli, U. et al. Orthostatic hypotension in de novo Parkinson disease. Arch. Neurol. 60, 1400-1404 (2003).

27. Bae, H. J., Cheon, S. M. \& Kim, J. W. Orthostatic hypotension in drug-naive patients with Parkinson's disease. J. Mov. Disord. 4, 33-37 (2011).

28. Gibb, W. \& Lees, A. The relevance of the Lewy body to the pathogenesis of idiopathic Parkinson's disease. J. Neurol. Neurosurg. Psychiatry 51, 745-752 (1988).

29. Postuma, R. B. et al. MDS clinical diagnostic criteria for Parkinson's disease. Mov. Disord. 30, 1591-1601 (2015).

30. Ryu, D. W. et al. Initial versus follow-up sequential myocardial 123I-MIBG scintigraphy to discriminate Parkinson disease from atypical Parkinsonian syndromes. Clin. Nucl. Med. 44, 282-288 (2019).

31. Chin, J. et al. Re-standardization of the Korean-instrumental activities of daily living (K-IADL): Clinical usefulness for various neurodegenerative diseases. Dement. Neurocogn. Disord. 17, 11-22 (2018).

32. Koh, S. B. et al. Validation of the korean-version of the nonmotor symptoms scale for Parkinson's disease. J. Clin. Neurol. 8, 276-283 (2012).

33. Ahn, Y. M. et al. A validation study of the Korean-version of the MontgomeryAsberg Depression Rating Scale. J. Korean Neuropsychiatr. Assoc. 44, 466-476 (2005).

34. Kwon, D. Y. et al. Translation and validation of the korean version of the 39-item Parkinson's disease questionnaire. J. Clin. Neurol. 9, 26-31 (2013).

35. Lee, S. A., Paek, J. H., Han, S. H. \& Ryu, H. U. The utility of a Korean version of the REM sleep behavior disorder screening questionnaire in patients with obstructive sleep apnea. J. Neurol. Sci. 358, 328-332 (2015).

36. Kaufmann, H., Malamut, R., Norcliffe-Kaufmann, L., Rosa, K. \& Freeman, R. The orthostatic hypotension questionnaire (OHQ): validation of a novel symptom assessment scale. Clin. Auton. Res. 22, 79-90 (2012).

37. Williams, B. et al. 2018 ESC/ESH guidelines for the management of arterial hypertension. Eur. Heart J. 39, 3021-3104 (2018).

38. Parati, G. et al. European Society of hypertension practice guidelines for ambulatory blood pressure monitoring. J. Hypertens. 32, 1359-1366 (2014).

39. Shin, N. Y. et al. Different functional and microstructural changes depending on duration of mild cognitive impairment in Parkinson Disease. AJNR Am. J. Neuroradiol. 37, 897-903 (2016).

40. Shin, N. Y. et al. Retrosplenial cortical thinning as a possible major contributor for cognitive impairment in HIV patients. Eur. Radiol. 27, 4721-4729 (2017).

41. Kim, J. S. et al. Automated 3-D extraction and evaluation of the inner and outer cortical surfaces using a Laplacian map and partial volume effect classification. Neuroimage 27, 210-221 (2005).

\section{ACKNOWLEDGEMENTS}

This research was supported by Basic Science Research Program through the National Research Foundation of Korea (NRF) funded by the Ministry of Science, ICT and Future Planning (NRF-2017R1D1A1B06028086).

\section{AUTHOR CONTRIBUTIONS}

S.-W.Y. and J.-S.K. designed the study; S.-W.Y., J.-S.K., J.-Y.Y., and K.-S.L. carried out data collection; E.Y., U.Y., and N.-Y.S. analyzed the MRI data; S.-W.Y. and J.-S.K. analyzed the clinical data; S.-W.Y. drafted the manuscript; S.-W.Y., J.-S.K., J.-Y.Y., E.Y., U.Y., N.-Y.S., and K.-S.L. revised the manuscript. J.-S.K. obtained funding. All authors read and approved the final version for publication.

\section{COMPETING INTERESTS}

The authors declare no competing interests.

\section{ADDITIONAL INFORMATION}

Supplementary information The online version contains supplementary material available at https://doi.org/10.1038/s41531-021-00181-y.

Correspondence and requests for materials should be addressed to J.-S.K.

Reprints and permission information is available at http://www.nature.com/ reprints

Publisher's note Springer Nature remains neutral with regard to jurisdictional claims in published maps and institutional affiliations.

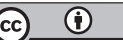

Open Access This article is licensed under a Creative Commons Attribution 4.0 International License, which permits use, sharing, adaptation, distribution and reproduction in any medium or format, as long as you give appropriate credit to the original author(s) and the source, provide a link to the Creative Commons license, and indicate if changes were made. The images or other third party material in this article are included in the article's Creative Commons license, unless indicated otherwise in a credit line to the material. If material is not included in the article's Creative Commons license and your intended use is not permitted by statutory regulation or exceeds the permitted use, you will need to obtain permission directly from the copyright holder. To view a copy of this license, visit http://creativecommons. org/licenses/by/4.0/.

(c) The Author(s) 2021 